\title{
Actividad física y uso de redes sociales en estudiantes de secundaria. Diferencias por sexo y grado escolar Physical activity and use of social media, in high school students. Differences by sex and school grade \\ 0 ddete Moreno-Muciño, Rosa Elena M edina Rodríguez, M aría Cristina Enríquez Reyna, Janet García \\ González, O swaldo Ceballos Gurrola \\ Universidad Autónoma de Nuevo León (México)
}

\begin{abstract}
Resumen. Las redes social es se han convertido en un fenómeno irreversible y de creciente uso entre niños y adolescentes. Esta utilización continuada puede conducir a un uso excesivo, que propicia el aumento del sedentarismo, un gran problema de salud pública. Por lo tanto, el objetivo de este estudio es analizar las diferencias por sexo y grado escolar al respecto de la actividad física y el uso de redes sociales en estudiantes de secundaria. La muestra estuvo conformada por 342 estudiantes de secundaria, 53.5\% mujeres y $46.5 \%$ hombres $(M=13.39 ; D E=0.84)$ de Nuevo León. Se aplicaron dos instrumentos, U sos y Gratificaciones de redes sociales en adolescentes mexicanos, e IPAQ -C. El 48\% de los estudiantes tiene bajo nivel de actividad física; $y$, aunque la intensidad se incrementa en los al umnos de mayor edad, disminuye la frecuencia en su prácticaa medidadel avanceescolar. Se registraron diferenciassignificativas en la conexión a redes social es, red favorita, dispositivo usado $(p<.01)$ en relación con el grado escolar; el 95.3\% tiene conexión a redes sociales, donde el $47.4 \%$ manifiestatener acceso a cualquier hora del día sin control parental, siendo los chicos y chicas del tercer grado quienes más tiempo pasan en esta actividad. Las redes más populares fueronYouTube y Facebook.
\end{abstract}

Palabras clave: actividad física, redes sociales, sedentarismo, adolescentes, hábitos.

\begin{abstract}
Social networks have become an irreversible and increasingly popular phenomenon among children and adolescents. This continued use can lead to excessive use, which is conducive to an increase in sedentary lifestyles, a major public health problem. Therefore, the aim of this study is to analyze the differences by sex and school grade regarding physical activity and the use of social networks in high school students. The sample consisted of 342 high school students, $53.5 \%$ female and $46.5 \%$ male $(M=13.39 ; S D=0.84)$ from Nuevo León. Two instruments, U ses and Gratifications of social networks in M exican adolescents, and IPAQ -C, were applied. Forty-eight percent of the students have a low level of physical activity; and although the intensity increases in older students, the frequency of physical activity decreases as school progresses. Significant differences were recorded in the connection to social networks, favorite network, device used $(p<.01)$ in relation to school grade; $95.3 \%$ have a connection to social networks, where $47.4 \%$ state having access at any time of the day without parental control, with boys and girls in the third grade spending more time on this activity. The most popular networks wereYouTube and Facebook.
\end{abstract}

Keywords: physical activity, social media, sedentary life, adolescents, habits.

\section{Introducción}

Las tecnologías de la información y las comunicaciones han generado en las últimas décadas cambios e innovacionesen la sociedad graciasasu interactividad (Jasso, López \& Díaz, 2017), han cambiado la forma de comunicación de las personas, así como la forma de relacionarse, llegando a formar parte de sus vidas (Villanueva Blasco \& Serrano-Bernal, 2019). La web se ha convertido en una herramienta de uso cotidiano para los adolescentes y las redes sociales en su instrumento más pre-

Fecha recepción: 05-12-20. Fecha de aceptación: 18-03-21

0 ddete M oreno Muciño

oddete.morenomc@uanl.edu.mx ciado (Pastor, Martín \& M ontes, 2019). El 90\% de los internautas mexicanos son usuarios de los móviles, lo que los posicionan como el dispositivo más popular. El $39 \%$ de los internautas mexicanos, está constituido por adolescentes y jóvenes (J asso, et al., 2017).

El acceso de los jóvenes a los dispositivos móviles, así como a las redes sociales es cada vez más temprano (Garitano, Garrido \& Andonegui, 2019; Guevara, U rchaga \& Sánchez-M oro, 2019; Pastor, et al., 2019). Estas plataformas que incluyen Facebook, YouTube, TikTok, Instagram, Pinterest, Twitter e incluso W hatsA pp se han hecho extremadamente populares en todo el mundo y son poderosos medios para llegar a miles de personas (Kubheka, Carter \& M waura, 2020). $L a s$ redes sociales se han convertido en un fenómeno 
irreversible en constante despliegue de numerosas posibilidades de interacción (Hefler et al., 2019; Hernández,Yanez \& Carrera2017), permitiéndolescrear, co-crear, modificar, compartir y participar con contenido generado por el usuario que sea fácilmente accesible (O 'Reilly et al., 2018). Su éxito se basa en su capacidad de adaptarse a los contextos sociales dinámicos de los usuarios y evolucionar con la sofisticación de la tecnología (Stellefson, Paige, Chaney \& Chaney, 2020). Aunque por sí solas no suponen un riesgo, el uso problemático se plantea cuando es casi ininterrumpido e impacta negativamente en el desarrollo normal de la vida cotidiana del adolescente (Díaz-Vicario, Mercader \& Gairín, 2019).

Sin embargo, pasar demasiadas horas frente a las pantal las disminuye el tiempo destinado a la práctica de ejercicio físico y relaciones sociales (Viner et al., 2019). Se ha reportado que en torno al $32 \%$ de los adolescentes muestran una frecuencia de conexión a internet elevada (más de cinco horas al día) (Villanueva-Blasco, et al. , 2019); por ende, estos cambios en el estilo de vida ocasionados por la tecnología han propiciado el aumento del sedentarismo (Vergara-Torres, et al. , 2020). Esta situación ha convertido la vida sedentaria en un factor de riesgo con grandes implicaciones para las políticas de salud pública (García et al., 2019). En este sentido, se sabe que la realización de actividad física (AF) de forma habitual por parte de los adolescentes, aporta grandes beneficios para la salud, como la prevención de enfermedades, el control del sobrepeso y la obesidad, mejoras en procesos depresivos y de estrés, beneficios psicológicos y sociales, entre otros (Fernández-Prieto, GinéGarriga, \& Canet, 2020). No obstante, al gunos resulta dos reportados en diversas investigaciones no son muy alentadores, ya que, se encuentra muy bajo porcentaje de actividad física en jóvenes, sobre todo en mujeres; hechos que no son exclusivos de una región o país, sino, se manifiesta a nivel global, en donde, por diferentes motivos, la baja incidencia de actividad física es muy frecuente (Chávez et al., 2018).

Por lo que, la planificación de la promoción de la salud debe tener en cuenta los conocimientos de los adolescentes sobre la importancia de la actividad física (Domínguez, López, \& Portela, 2018), ya que, la prescripción y realización de actividad física, se convierte en intervención fundamental como factor protector para disminuir el riesgo para otras enfermedades crónicas (García et al, 2019). De esta manera, con una tasa de penetración tan alta (Huo, et al. , 2019); las redes sociales presentan una oportunidad significativa para mejorar los programas y campañas de promoción a la salud y, apoyar a la salud pública (Kubheka, et al., 2020).

U na vez expuesto lo anterior, se plantea como objetivo de estudio analizar las diferencias por sexo y grado escolar al respecto de la actividad física y el uso de redes sociales en estudiantes de secundaria. Con base en la revisión de la literatura se pusieron a prueba las siguientes hipótesis de investigación: $\mathrm{H}_{\mathrm{a}}$ : a mayor edad menos actividad física; $\mathrm{H}_{2}$ : a mayor uso de redes sociales menos actividad física.

\section{Método}

\section{Tipo de estudio}

Se trata de una investigación de enfoque cuantitativo no experimental de diseño transversal descriptivo, comparativo y correlacional (Hernández, Fernández \& Baptista, 2014) sobre el nivel de actividad física y el uso de redes sociales en adolescentes mexicanos.

\section{Participantes}

Participa una muestra no probabilistica de 342 estudiantes de educación secundaria que asisten a dos escuelas públicas del municipio de San N icolás de los Garza, Nuevo León, México; 183 mujeres(53.5\%) y 159 hombres (46.5\%), con un rango de edad entre 12 y 16 años $(13.39 \pm 0.84)$, de primero $(n=71)$, segundo $(n=91)$ y tercer grado escolar $(n=180)$, de participación voluntaria corroborada con consentimiento informado por parte del padre de familia y asentimiento de los participantes.

\section{Instrumentos}

Se utilizó el cuestionario de U sos y Gratificaciones de redes sociales en adolescentes; este instrumento se compone de 24 ítems dividido en dos apartados: el primero utilizó una escala tipo Likert, donde uno es nunca y cinco essiempre, con 14 ítems con un alfade C ronbach $=.84$, de buena consistencia interna; con un índice de KMO de 0.841, y prueba de Bartlett estadísticamente significativa $\left(\div^{2}=876.54, g l=91 ; p<.000\right)$. Se extra jeron 4 factores con valores Eigen de 1 o mayores que, 
conjuntamente, explican un $52.65 \%$ de la varianza total. Se agrupan cuatro factores: Presencia social $(22,16$, 20, 18, 21, 17, 23), Evasión (15, 14), Entretenimiento $(12,13,19)$, Auto-presentación $(24,25)$ (Ver tabla 1).

En Evasión, que se caracteriza por eludir una realidad, se hacen cuestiones como «uso las redes sociales como la mejor manera de evadirme del mundo». Para la Presencia Social, la cual, es el grado en que una persona es percibida por los demás miembros de una comunidad virtual (Gutiérrez-Santiuste $\&$ GallegoArrufat, 2017), se indagan aspectos como «soy capaz de ser yo mismo y mostrar qué clase de persona soy». Referente al Entretenimiento o lo que es lo mismo distracción, se utilizan oraciones como «uso redes sociales cuando he tenido un mal día», y, finalmente preguntas como «uso redes sociales para demostrar a otros que soy el mejor», fueron empleadas para el factor Auto Presentación, que está relacionada con la apariencia física (por ejemplo, estar a la moda o lucir saludable) y las actividades sociales (Pérez, 2018).

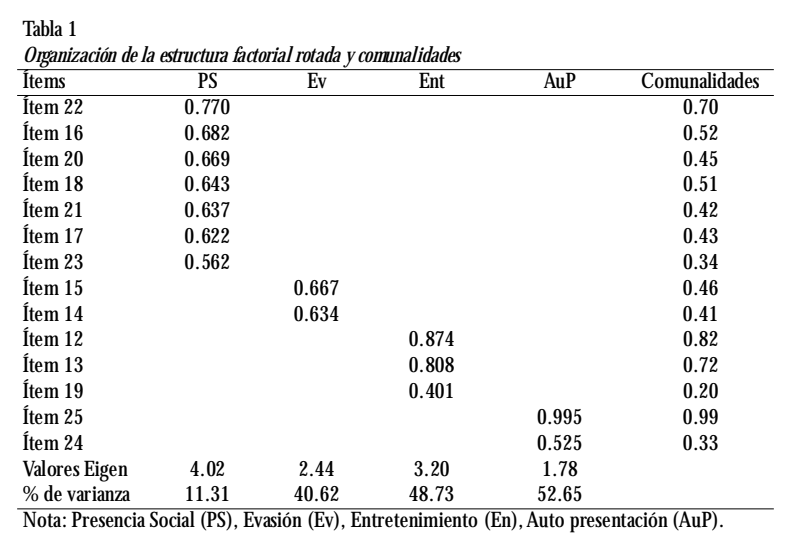

Los 10 ítems restantes del segundo apartado hacen referencia a las características de las redes sociales y conexión a dispositivos móviles: conexión a redes sociales, red social favorita, uso de dipositivos, tiempo de conexión, uso de videojuegos y aplicaciones que activan a los adolescentes; tema de interés en esta publicación.

El segundo instrumento fue el «Cuestionario Internacional de Actividad Física para niños» (IPAQ -C), con valoresapropiados de confiabilidad conAlfade Cronbach de 0.83 y KM O $=.877$ (Crocker et al., 1997; Manchola González, Bagur-Calafat \& Girabent-Farrés, 2017). Diseñado para medir actividad física bajo, intermedio y al to semanal en niñosy adolescentes (Sallis, et al ., 1991) que consiste en diez ítems con opción de respuesta del uno al cinco, donde a mayor puntuación mayor nivel de $\mathrm{AF}$.
El primer ítem resume el promedio obtenido a partir de un listado de actividades deportivas en el que el participante eligió la frecuencia con la que realiza cada una de esas actividades; las preguntas dos a ocho, cuestionan sobre las actividades realizadas en periodos específicos del día y de la semana, tanto en la escuela como fuera de ella. La novena pregunta cuestiona sobre la autoevaluación de actividad física por cada uno de los días de la semana. La última pregunta, cuestiona sobre al guna enfermedad u otro acontecimiento que impidió que el estudiante realice sus actividades regulares en la última semana. Se recomienda obtener el promedio de respuesta del listado de actividades preliminar y de los días de la semana (pregunta nueve) y estimar un promedio de respuesta de los nueve ítems para obtener la puntuación global del cuestionario (IPAQ-C) y, por ende, los niveles de AF.

\section{Procedimiento}

La aplicación de los instrumentos se realizó mediante encuestas electrónicas en la sala de cómputo de cada institución, con un estimado de 15 minutos por alumno para responder; siempre bajo el cuidado y observación en todo momento por encuestadores entrenados. A los padres de familia de los estudiantes, se les solicitó la firma de un formato de consentimiento informado detallando el objetivo de la investigación, proporcionando, además, el aviso de privacidad para manejo de datos personales; el proyecto fue revisado y aprobado por la Coordinación de Investigación y Ética de la Facultad de Organización Deportiva de la Universidad Autónoma de Nuevo León (REPRIN-FOD-68). A todos los voluntarios se les dió a conocer el objetivo del estudio, se solicitó consentimiento informado y se explicó la forma de contestar el instrumento. Se aclaró que la encuesta era anónima y para uso académico de investigación exclusivamente.

\section{Análisis de datos}

Se utilizó el paquete estadístico Statistical Package for the Social Sciences (SPSS) versión 24 para los análisis. Se realizó estadística descriptiva y estadística inferencial. La normalidad de los datos se comprobó mediante la prueba de Kolmogorov-Smirnov, utilizando la corrección de Lilliefors. Tomando en cuenta la escala de medición de las variables con distribución no normal se aplicaron pruebas de U de Mann-Withney, 
Kruskal-Wallis y análisis de correlación para las pruebas de hipótesis considerando un valor crítico de alfa de. 05 .

\section{Resultados}

La muestra estuvo constituida por 342 estudiantes deeducación secundaria, 20.8\% de primer grado, 26.6\% de segundo y $52.6 \%$ de tercero; con edad promedio de 13.39 años ( $D E=0.84$ ); el $49.4 \%$ refirió tener 14 años; del total de la muestra, $53.5 \%$ son mujeres y $46.5 \%$ hombres.

En relación con la práctica de actividad física, los resultados que se obtienen son que, el $50.3 \%$ realiza actividades que son consideradas de bajo nivel, el 49.1\% de nivel intermedio y solamente el $6 \%$ reporta tener actividades clasificadas como de alto nivel. Aunado a lo anterior, se encuentra una disminución en el nivel de actividad física en los estudiantes de grado escolar avanzado, siendo los hombres quienes se mantienen menos activos (50.5\% de las mujeres por $57.5 \%$ de hombres); sin embargo, a pesar observarse una disminución en la frecuencia de la práctica de ejercicio físico, es al mismo tiempo, la etapa donde la intensidad se incrementa (ver tabla 2). En la escuela, sólo el 19.6\% refirió tener clases constantes de educación física en las que realizan actividades de intensidad moderada a vigorosa; el $13.5 \%$ señaló que no se le impartía la asignatura. El $63.2 \%$ de los estudiantes manifiesta tener actividades ligeras que les permiten recorrer espacios pequeños en el descanso dentro de lainstitución educativa; $y$, tanto hombrescomo mujeres reportan esta situación como preferente.

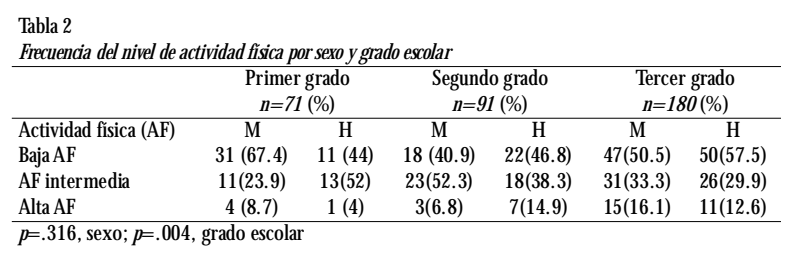

Respecto a las actividades que se realizan en el receso escolar, son los alumnos del tercer año, quienes permanecen más tiempo sentados durante este lapso $(16.7 \%)$ y son las mujeres quienes más reportaron esta actividad (13.7\%). Los participantes que se mantienen más activos son las mujeres (5.9\%) del segundo año escolar (4.4\%) quienes corren o juegan durante el descanso escolar.

En referencia a la cantidad de ejercicio físico practi- cado por las tardes, la mayoría de los participantes se mantiene activo unaveza lasemana; en cambio, el 28.1\% comentó que durante los fines de semana estaban activos dos o tres veces en este periodo; siendo las mujeres $(8.5 \%)$ quienes se mantienen practicando actividad física más de 6 veces a la semana en comparación con los hombres $(6.7 \%)$. Respecto al grado escolar son los participantes del tercer grado quienes muestran más actividades sedentarias (20.5\%) manteniéndose activos solamente una vez a la semana por las tardes.

En lo que, al uso de redes social es se refiere el $95.3 \%$ tiene conexión a ellas y emplean una importante cantidad de tiempo al día para hacerlo (el $47.4 \%$ manifiesta tener acceso a cualquier hora), empleando el teléfono móvil para tal efecto (82.2\%). YouTube con 36.0\% y Facebook con 33.3\% son las redes sociales más populares (Tabla 3). Además, se obtuvieron datos relacionados al uso de videojuegos y aplicaciones que les motivaran a realizar actividad física de manera regular. El 34.5\% juegan videojuegos toda la semana, con más de una hora de conexión (35.4\%), sin embargo, el $71.9 \%$ de los alumnos que tienen videojuegos, no cuentan con al guno que incentive el ejercicio físico; dato similar a las aplicaciones usadas por los estudiantes, el $75.4 \%$ de quienes las usan no lo hacen para monitorear o registrar su actividad física.

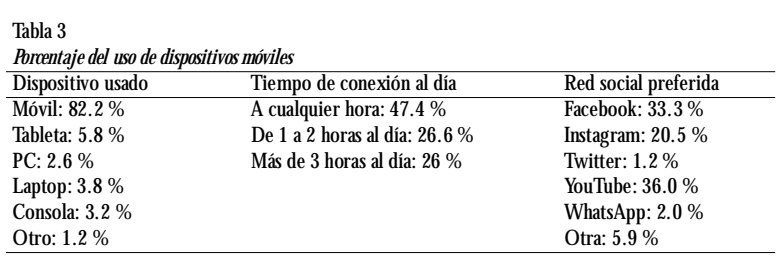

La tabla 4 muestra que, al comparar las variables de estudio por grado escolar, se registraron diferencias significativas en la conexión a redes sociales, red favorita, dispositivo usado ( $p<.01$ ), así como el tiempo que permanecen conectados y aplicaciones que les permite activarse físicamente $(p<.05)$; no así, la actividad física medida por el IPAQ -C y el uso de videojuegos que activan. Los datos se presentan con frecuencia y porcentaje y no se distinguen diferencias por sexo (H-M).

Las variables observadas no mostraron diferencias por sexo en relación a la actividad física; y, a más edad y grado escolar, mayor es el uso de las redes sociales, ya que se observa una asociación negativa. En el resultado global del IPAQ-C, las mujeres del segundo grado son 
Tabla 4

Características descriptivas del nivel de actividad física y uso de redes sociales según por grado escolar y sexo de los estudiantes.

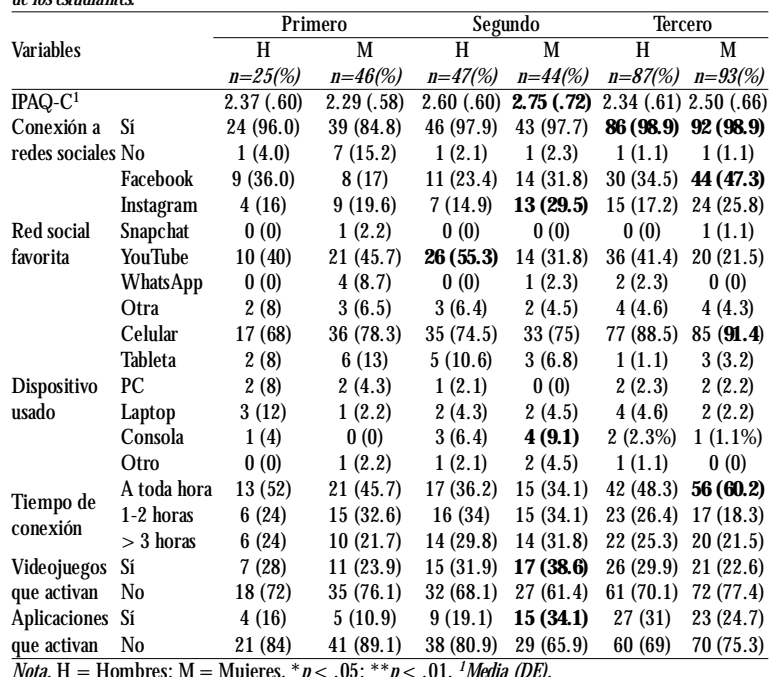

quienes mayor cantidad de actividad física a la semana reportan $(M=2.75, D E=0.72)$. Referentealaconexión y redes sociales, no se observan diferencias importantes entre el sexo y el grado escolar de los participantes. El uso de redes sociales es mayor en mujeres de tercer grado (47.3\%) para Facebook, mujeres de segundo gra do $(29.5 \%)$ para Instagram y hombres de segundo gra do (55.3\%) paraYouTube; y son también las mujeres del tercer año, quienes más reportan el uso de móviles (91.4\%). De igual manera, las chicas del último grado manifiestan tener conexión a cualquier hora del día, sin restricción de tiempo $(60.2 \%)$, y, por último, con respecto al uso de videojuegos y aplicaciones que les permitieran mantenerse en actividad física continua, son las mujeres de segundo grado quienes más las usan (38.6\% y $34.1 \%$, respectivamente) (Ver tabla 5).

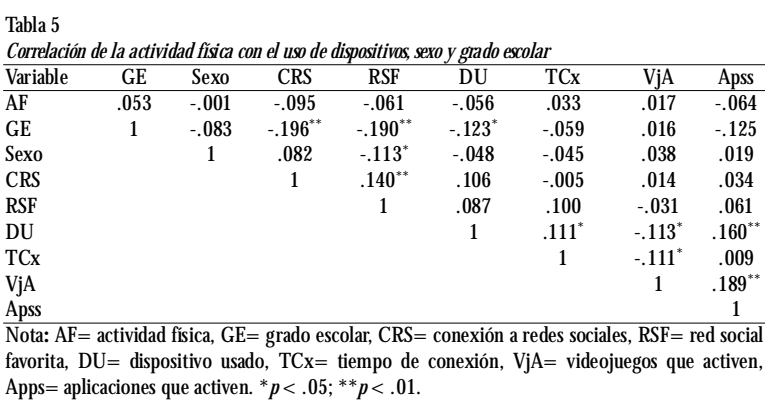

La descripción detallada del promedio de respuesta a cada una de las preguntas del Cuestionario IPAQ -C sobre las características de la actividad física reportada por los participantes, se presenta en la tabla 6. En el listado de actividades deportivas semanales, la media de días que se realiza alguna de ellas es de 1.59 días a la semana ( $D E=0.39$ ), siendo las mujeres quienes más ejercicio reportaron $(M=1.69, D E=0.43$ para las mujeres y $M=1.49, D E=0.30$ para hombres); donde se cuestiona sobre actividades como saltar la cuerda, andar en bicicleta, nadar, jugar fútbol 0 baloncesto, artes marciales, entre otras. Los valores más altos en hombres se observaron al respecto de actividades posteriores a sus clases del colegio; en contraste, para las mujeres los valores más altos se presentaron en la clase de educa ción física. Finalmente, como se mencionó con anterioridad, se debe obtener el promedio de los nueve ítems con el que se adquiere la puntuación global del cuestionario (actividad física realizada en los últimos siete días), resultando $M=2.47$ días a la semana $(D E=0.65)$ entre el ejercicio realizado en la escuela, en fines de semana y por las tardes.

\begin{tabular}{|c|c|c|c|c|c|c|c|}
\hline \multirow{2}{*}{\multicolumn{3}{|c|}{$\begin{array}{c}\text { Todos } \\
(n=342) \\
\text { Media } \quad D E\end{array}$}} & \multicolumn{2}{|c|}{$\begin{array}{l}\text { Hombres } \\
(n=159)\end{array}$} & \multicolumn{2}{|c|}{$\begin{array}{c}\text { Mujeres } \\
(n=183)\end{array}$} & \multirow[b]{2}{*}{$p$} \\
\hline & & & Media & $D E$ & Media & $D E$ & \\
\hline 1. LAD & 1.59 & 0.39 & 1.49 & 0.30 & 1.69 & 0.43 & .014 \\
\hline 2. Clase Educación Física & 3.12 & 1.34 & 3.09 & 1.39 & 3.14 & 1.30 & .513 \\
\hline 3. Receso matutino & 1.96 & 0.78 & 1.86 & 0.53 & 2.05 & 0.93 & .002 \\
\hline 4. Comida & 1.60 & 0.89 & 1.57 & 0.77 & 1.63 & 0.99 & .146 \\
\hline 5. Post-clases & 3.10 & 1.29 & 3.14 & 1.35 & 3.07 & 1.24 & .402 \\
\hline 6. Actividad vespertina & 2.92 & 1.32 & 3.05 & 1.25 & 2.81 & 1.37 & .000 \\
\hline 7. Fin de semana & 2.73 & 1.20 & 2.56 & 1.18 & 2.87 & 1.21 & .202 \\
\hline 8. Descripción per sonal & 2.45 & 1.18 & 2.44 & 1.18 & 2.46 & 1.18 & .202 \\
\hline 9. Resumen semanal & 2.74 & 0.82 & 2.62 & 0.77 & 2.83 & 0.86 & .204 \\
\hline IPAQ -C & 2.47 & 0.65 & 2.42 & 0.61 & 2.51 & 0.67 & .277 \\
\hline
\end{tabular}

\section{Discusión}

El propósito de este estudio fue analizar la actividad física y el uso de redes sociales en estudiantes de secundaria; diferencias por sexo y grado escolar. Además de los resultados expuestos en el presente, también se obtuvieron respuestas de la principal barrera para la práctica de actividad física, destacando la falta de tiempo como respuesta número uno; lo que puede estar rela cionado a lo encontrado en esta investigación, pues la cantidad de horas en pantalla que pasan los adolescentes con un dispositivo móvil yl o conectados a redes sociz les y/ o con consolas de vídeo juegos; merma considera blemente la práctica de actividad física recurrente (Van Woudenberg et al., 2020).

Los resultados relacionados a la actividad física, coinciden con algunos otros estudios ya que se observa que el nivel de actividad física en adolescentes es bajo y además, disminuye conforme a la edad, lo cual permite aceptar la $\mathrm{H}_{1}$ (Beltrán-Carrillo, Devís-Devís \& Peiró- 
Velert, 2012; Berglind \& Tynelius, 2018; De Baere, Lefevre, De M artelaer, Philippaerts \& Seghers, 2015; Guevara, U rchaga \& Sánchez-M oro, 2019; Kettner, et al., 2013; Meneses \& Ruiz, 2017; Richmond, Smith, Ness, den Hoed, M cM ahon \& Timpson, 2014), lo que se ha manifestado desde hace varios años; $y$, aunque la intensidad se incrementa en los alumnos de cursos superiores, disminuye la frecuencia de actividad física a medida del avance escolar (Franco-Arévalo, Feu $\&$ De la Cruz-Sánchez, 2020; Garcinuño, García, Alonso \& López, 2011; Zueck-Enríquez, Ramírez-García, Rodríguez-Villalobos \& Irigoyen-Gutiérrez, 2020), y se relaciona de manera importante con el uso desmedido de dispositivos móviles (Berglind \& Tynelius, 2018; De Baere, et al., 2015; Guevara, U rchaga \& SánchezM oro, 2019;Twenge \& Campbell, 2018;Twenge, Krizan $\&$ Hisler, 2017).Y, por si fuera poco, las horas clase de educación física se reportan disminuidas o nulas 0 de baja intensidad en el caso de las que sí son impartidas. Por lo que, cobran vital importancia al convertirse en el único espacio y momento en el día, en el que el adolescente puede realizar ejercicio físico (González, Gómez-López \& Granero-Gallegos, 2019).

Por otro lado, en lo referente al uso de redes socia les, los resultados coinciden con otros autores al reportar a Facebook, YouTube e Instagram como las preferidas entre los adolescentes (Calderón-Hernández, Ma drigal-Moreno \& Madrigal-M oreno, 2019; Hanna, 0 campo, Janna, Mena \& Torreglosa, 2020; MacedoRouet, Salmerón, Ros, Pérez, Stadtler \& Rouet, 2020; Tejada, Castaño \& Romero, 2019) y, sin importar la edad o el grado escolar, todos tienen acceso a ellas con escaso control parental. Así, se incrementa la importancia del contenido ofrecido en estas plataformas y que incluso, sumado con el uso de videojuegos pueden ayudar a reforzar y aumentar su autoestima y autoconcepto, cuando superan las dificultades que se les presentan (Sánchez-Zafra, Ramirez-Granizo, Baez-Mirón, Moreno-Arrebola \& Fernández-Revelles, 2019). Tema para considerar de manera muy puntual puesto que, la motivación principal para la práctica de ejercicio físico en la población estudiada fue la intrínseca, que es la motiva ción que tiene una relación directa y positiva con las necesidades de competencia y autonomía, pues a mayor nivel de motivación, mayor es el rendimiento obtenido (García, et al., 2019).Y ocurre una situación similar con las aplicaciones comerciales, pues se ha identificado un incremento en la actividad física realizada a partir de su uso recurrente (Vergara-Torres, et al., 2020); en el presente estudio, se observó una disminución de la actividad física y un incremento del uso de redes sociales, lo cual lleva a aceptar la $\mathrm{H}_{2}$.

De tal forma que, resulta apremiante hacer intervenciones educativas en los jóvenes en temas de estilos de vida sal udable y práctica de la actividad física (ZueckEnríquez, et al., 2020). Las redes sociales y las tecnologías de la información y comunicación pueden proponerse iniciativas que hagan uso de su popularidad entre los jóvenes con la promoción de prácticas saludables. Entre otras actividades, se puede citar el cómo utilizar una aplicación móvil para búsqueda de información basada en evidencias, realizar un vídeo para utilizarlo en promoción de salud, recomendación del uso de la web con información fiable sobre salud, o contactar con otros profesionales a través de una red social (Fernández $\&$ Lafuente, 2016).

El rápido desarrollo de los medios de comunicación social ha hecho que aumente su utilización por parte de los niños y adolescentes con fines de salud y bienestar (Bopp \& Stellefson, 2020). Encontrar el equilibrio entre costo y beneficio es el principal reto; el empleo de los medios tradicionales en promoción en salud implica altos costos y planificar una campaña con resultados similares en redes sociales, los reduce significativamente (Acevedo, Toctaguano \& Troya, 2019).

Es conocido que el ejercicio físico se asocia a múltiples beneficios para la salud (Cortés-Cortés, Alfaro, M artínez \& Veloso, 2019; González, et al. , 2019; O rganización M undial delaSalud [OMS], 2018); de estemodo, la necesidad de incrementar la actividad física se está convirtiendo en una prioridad político-social y, sobre todo, impul sarla desde la edades tempranas (Rodríguez, García \& Minguet, 2020), por lo que el mensaje y el contenido del mismo, toman singular importancia en la promoción de hábitos sanos.

Los usuarios jóvenes han informado sobre los beneficios del uso de ciertas plataformas en áreas que van desde el aprendizaje, la socialización, mayores niveles de apoyo social y emocional, y la creatividad (Goodyear \& Armour, 2019). Algunos departamentos de salud a nivel mundial tienen presencia considerable en redes sociales, pero con mínimo contenido en promoción ala 
salud (Kubheka, et al. ., 2020). Las campañas de los medios de comunicación de masas no son una estrategia eficaz para promover la actividad física en jóvenes; hoy en día tienen menos probabilidades de utilizar los medios de comunicación tradicionales que los medios sociales en línea. El uso de los medios social es como plataforma de aprendizaje requiere una mayor evaluación para comprender mejor las preferencias de los adolescentes y la forma en que se relacionan con la informa ción en línea (0 'Reilly, et al., 2018; Van Woudenberg et al., 2020).

La aplicación de tecnologías en la salud puede aumentar la capacidad de adquirir y distribuir informa ción, automatizar el análisis y aumentar la transparencia en los procesos de promoción de la salud (Conrad, et al., 2020). Se ha observado que el uso de las redes sociales para el intercambio de información médica casi se había duplicado en un año (2013: 2.0\%; 2014: 3.8\%) (Huo, et al., 2019). Estas plataformas proporcionan vehículos parala comunicación y laautoexpresión que ofrecen a los jóvenes una oportunidad sin precedentes para aprender sobre temas de salud, compartir sus conocimientos e ideas y obtener el apoyo de sus pares (Halsall, Garinger, Dixon \& Forneris, 2019).

Las limitaciones que se presentaron en este estudio fueron que, no se indagó sobre el contenido específico revisado en los dispositivos móviles ni en redes socia les, lo que permitiría en estudios futuros, enfocar las estrategias de intervención en materia de salud pública, con base en los intereses del usuario.

\section{Conclusiones}

La práctica de actividad física por parte de los adolescentes que participaron en este estudio, no cubre las recomendaciones mínimas internacionales de 60 minutosal día de intensidad moderada a vigorosa; por lo que, fomentar la autonomía en su realización para ser una actividad prioritaria en el tiempo libre, es fundamental; sin olvidar la gran importancia que tiene la realización de ejercicio físico en las instituciones educativas.

$Y$, ya que, el uso de tecnologías de la información y comunicación, las redes sociales y los sitios Web que comparten vídeos son cada vez mayores en poblaciones jóvenes, las estrategias de marketing digital pueden y deben ser usadas en la promoción de actividad física para lograr impactar en el desarrollo de los hábitos saludables en la población en general y en los adolescentes y niños específicamente; donde las iniciativas públicas y legislaciones ayuden a reducir los costos que puedan generar campañas de esta naturaleza, sin dejar de lado el vínculo con la iniciativa privada para asegurar mayor efecto.

Se ha podido comprobar que la actividad física que realizan los estudiantes de educación secundaria disminuye conforme a la edad y el uso de redes sociales, siendo un dato preocupante para la salud futura de esta población.

Los gobiernos pueden usar losmedios digital escomo una nueva fuente de información que les permita comprender las necesidades y comportamientos de las personas usándolas para la atención médica. Potencialmente, las intervenciones pueden ser más efectivas cuando utilizan el impacto de otros adolescentes practicando actividad física y recibiendo mensajes que son comunicados a través de las redes sociales y laWeb, donde los centros educativos y la clase de educación física pueden ser una alternativa eficaz.

La integración y utilización de lasTIC en la salud y los cuidados es un reto inmediato que se debe afrontar ofreciendo una manera diferente de interacción donde impera la acción en grupos de edades tempranas para prevenir y revertir hábitos insanos de manera más eficaz.

\section{Referencias}

Acevedo, D., Toctaguano, S. \& Troya, C. (2019). Impacto de Facebook en la promoción de salud en Santo Domingo de losT sáchilas-Ecuador. Práctica Familiar Rural, 4(2). https:/ / doi.org/ 10.23936/ pfr. v4i2.74

Franco-Arévalo, D., Feu, S. \& De la Cruz Sánchez, E. (2020). Diferencias entre el medio rural y urbano en el nivel de actividad física en la transición de la educación primaria a la educación secundaria. Revista Especializada en Salud Pública, 94(29), e202005026. Beltrán-Carrillo, V. J., Devís-D evís, J., \& Peiró-Velert, C. (2012). Actividad física y sedentarismo en adolescentes de la Comunidad Valenciana. Revista Internacional de Medicina y Ciencias de la Actividad Física y del Deporte, 12(45), 123-137. Http:// 
cdeporte. rediris. es/ revista/ revista45/ artactividad266.htm

Berglind, D., \&Tynelius, P. (2018). O bjectively measured physical activity patterns, sedentary time and parentreported screen-time across the day in four-yearold Swedish children. BMC Public H ealth, $18(1), 69$. http:/ / doi.org/ 10.1186/ s12889-017-4600-5

Bopp, T., \& Stellefson, M. (2020). Practical and Ethical Considerations for Schools Using Social Media to Promote Physical Literacy in Youth. International Journal of Environmental Research and Public Health, 17 (4), 1225. http:/ / doi.org/ 10.3390/ ijerph17041225

Calderón-Hernández, A., Madrigal-M oreno, S., \& Ma drigal-M oreno, F. (2019). Facebook Como Espacio de Expresión Emocional de los Adolescentes en Morelia, México. European Scientific Journal, 15(16), 1-14. http:/ / dx. doi.org/ 10.19044/ esj.2019.v15n16p1

Chávez, V. M. E., Salazar, C. M., Hoyos, R. G., Bautista, J. A., González, G. L. \& 0 garrio, P. C. E. (2018). Actividad física y enfermedades crónicas no transmisibles de estudiantes mexicanos en función del género. Retos: nuevas tendencias en educación física, de portey recreación, (33)169-174.

Conrad, E. J., Becker, M., Powell, B., \& Hall, K. C. (2020). Improving health promotion through the integration of technology, crowdsourcing, and social media. Health promotion practice, 21(2), 228-237. http:/ / doi.org/ 10.1177/ 1524839918811152

Cortés-Cortés, M. E., Alfaro Silva, A., Martínez, V., \& Veloso, B. C. (2019). Desarrollo cerebral y aprendizaje en adolescentes: Importancia de la actividad física. Revi sta médica de Chile, 147(1), 130-131. http:/ / dx.doi.org/ 10.4067/ S0034-98872019000100130

Crocker, P. R., Bailey, D. A., Faulkner, R. A., Kowalski, K. C., \& M cGrath, R. (1997). Measuring general levels of physical activity: preliminary evidence for the Physical Activity Q uestionnaire for O Ider Children. Medicine and science in sports and exercise, 29(10), 1344-1349.

Cronbach, L.J. (1951). Coefficient al pha and theinternal structure of tests. Psychometrika, 16, 1-16. https:/ / bit.ly/ 3gBEleH

De Baere, S., Lefevre, J., De Martelaer, K., Philippaerts, R., \& Seghers, J. (2015). Temporal patterns of physical activity and sedentary behavior in 10-14 year-old children on weekdays. BMC public health, 15(1), 791. http:/ / doi.rog/ 10.1186/ s12889015-2093-7

Díaz-Vicario, A., Mercader Juan, C., \& Gairín Sallán, J. (2019). U so problemático de las TIC en adolescentes. Revista electrónica de investigación educativa, 21(1), 1-11. https:/ / doi. org/ 10.24320/ redie. 2019.21.e07.1882

Domínguez, A. J., López, C. A., \& Portela, P. I. (2018). Validación del autoinforme de motivos para la práctica del ejercicio físico con adolescentes (AMPEF): diferencias por género, edad y ciclo escolar. Retos: nuevas tendencias en educación física, deportey recreación, (33), 273-278.

Fernández, S. S. \& Lafuente, R. N. (2016). Integración de internet y las redes sociales en las estrategias de salud. Enferm Clin, 26(5). http:/ / dx.doi.org/ 10.1016/ j.enfcli.2016.08.001

Fernández-Prieto, I., Giné-Garriga, M ., \& CanetVélez, 0. (2020). Barreras y motivaciones percibidas por adolescentes en relación con la actividad física. Estudio cualitativo a través de grupos de discusión. Revista Española de Salud Pública, 93(12).

García, J. E. M., Palmero, J. R., García, S. L., Guillamón, A. R., Soto, J. J. P., \& Cantó, E. G. (2019). Propiedades psicométricas de la Escala de Motivación Deportiva y análisis de la motivación en las clases de educación física y su relación con nivel de práctica de actividad física extræescolar. Retos: nuevas tendenciasen educación física, deportey recreación, 36, 283-289. García, S. J. M., Delgado, J. C., Moreira, M. C. C., Soriano, A. B. C., Andrade, F. A. B., \& Villacreses, M. L. E. (2019). Prevención de paciente con problemas de sedentarismo cardiovascular. Dominio de las Ciencias, 5(1), 32-53. http:/ / dx. doi.org/ 10.23857/ dc.v5i1.849

García-Ruiz, R., Ruiz, R.G., \& Gómez, Á.H. (2018). Redes sociales y estudiantes: motivos de uso y gratificaciones. Evidencias parael aprendizaje. Aula Abierta, 47(3), 291-298. https:/ / bit. ly/ 2ZCKN d0

Garcinuño, A. C., García, I. P., Alonso, I. C., \& López, S. A. (2011). Determinantes del nivel de actividad física en escolares y adolescentes: estudio O PACA. Anales de Pediatría, 74, (1), 15-24. http:/ / doi. org/ 10.1016/ j. anpedi.2010.08.009 
Garitano, E. T., Garrido, C. C., \& Andonegui, A. R. (2019). Los hábitos de uso en las redes sociales de lospreadolescentes. RIED. Revista Iberoamericana deEducación a Distancia, 22(2), 119-133. http:/ / dx. doi.org/ 10.5944/ ried.22.2.23245

Guevara, R. M., U rchaga, J. D., \& Sánchez-Moro, E. (2019). Horas de pantalla y actividad física de los estudiantes de Educación Secundaria. Eur. J. Health Res, 5, 133-143. http:/ / doi.org/ 10.30552/ ejhr.v5i2. 184

Gutiérrez-Santiuste, E., \& Gallego-Arrufat, M. J. (2017). Presencia social en un ambiente colaborativo virtual de aprendizaje: análisis de una comunidad orientada a la indagación. Revista mexicana de investigación educativa, 22(75), 1169-1186.

González, V. M., Gómez-López, M., \& Granero-Gallegos, A. (2019). Relación entre la satisfacción con las clases de Educación Física, su importancia y utilidad y la intención de práctica del alumnado de Educa ción Secundaria O bligatoria. Revista Complutense de Educación, 30 (2), 479. http:/ / dx. doi. org/ 10.5209/ RCED.57678

González-Vázquez, A., \& Igartua, J.J. (2018) ¿Por qué los adolescentes juegan videojuegos? Propuesta de una escala de motivos para jugar videojuegos a partir de la teoría de usos y gratificaciones. Cuadernos (42), 135-146. http:/ / doi.org/ 10.7764/ cdi.42.1314

Goodyear, V. A., \& Armour, K. M. (2019). Young people, social media and health (p. 232). Taylor \& Francis.

Halsall, T., Garinger, C., Dixon, K., \& Forneris, T. (2019). Evaluation of a social media strategy to promote mental health literacy and help-seeking in youth. Journal of Consumer $H$ ealth on the Internet, 23(1), 13-38.

https: / / doi.org/ 10.1080/ 15398285.2019.1571301

Hefler, M., Kerrigan, V., Henryks, J., Freeman, B., \& Thomas, D. P. (2019). Social media and health information sharing among Australian Indigenous people. Health Promotion International, 34(4), 706-715. http:/ / doi. org/ 10.1093/ heapro/ day018

Hernández Mite, K. D. , Yanez Palacios, J. F., \& Carrera Rivera, A. A. (2017). Las redes sociales y adolescencias: repercusión en la actividad física. Revista universidad y sociedad, 9 (2), 242-247.

Hernández, S., Fernández, C., \& Baptista, L. (2014). M etodología dela Investigación. M éxico: M c Graw Hill.
Hanna, M. I., O campo, M. M., Janna, N. M., Mena, M. C., \& Torreglosa, L. D. (2020). Redes sociales y calidad de vida relacionada con la salud en estudiantes universitarios. Revista Cuidados.11(1): e953. http:/ / dx. doi.org/ 10.15649/ cuidarte. 953

Huo, J., Desai, R., Hong,Y. R., Turner, K., Mainous III, A. G. \& Bian, J. (2019). U se of social media in health communication: findings from the $\mathrm{Health}$ Information National TrendsSurvey 2013, 2014, and 2017. Cancer Control, 26(1). http:/ / doi.org/ 10.1177/ 1073274819841442

Jasso, M. J. L., López, R. F. L., \& Díaz, L. R. (2017). Conducta adictiva a las redes sociales y su relación con el uso problemático del móvil. Acta de investigación psicológica, 7(3), 2832-2838. https:/ / doi.org/ 10.1016/ j.aipprr.2017.11.001

Kettner, S., Kobel, S., Fischbach, N., Drenowatz, C., D reyhaupt, J., W irt, T., .. \& \& Steinacker, J. M . (2013). $O$ bjectively determined physical activity levels of primary school children in south-west Germany. BMC Public Health, 13(1), 895. http:// www. biomedcentral.com/ 1471-2458/ 13/ 895

Kubheka, B. Z., Carter, V. \& \& M waura, J. (2020). Social media health promotion in South Africa: Opportunities and challenges. African Journal of Primary H ealth Care \& Family M edicine, 12 (1). https: / / doi.org/ 10.4102/ phcfm.v12i1.2389

Macedo-Rouet, M., Salmerón, L., Ros, C., Pérez, A., Stadtler, M., \& Rouet, J. F. (2020). Are frequent users of social network sites good information evaluators? An investigation of adolescents' sourcing abilities. Journal for the Study of Education and D evelopment, 43(1), 101-138. https:/ / doi.org/ 10.1080/02103702.2019.1690849

M anchola-González, J., Bagur-Calafat, C., \& GirabentFarrés, M. (2017). Fiabilidad de la versión española del Cuestionario de actividad física PAQ -C / Reliability of the Spanish Version of Q uestionnaire of Physical Activity PAQ -C. Revista Internacional De Medicina y Ciencias de la Actividad Física y del Deporte, 17(65), 139-152. https:/ / doi.org/ 10.15366/ rimcafd2017.65.010

Meneses, M. , \& Ruiz, J. F. (2017). Estudio longitudinal de los comportamientos y el nivel de actividad físico-deportiva en el tiempo libre en estudiantes de Costa Rica, M éxico y España. Retos, nuevas tendencias 
en educación física, deportey recreación, 31, 219-226.

O rganización M undial de la Salud [O M S]. (2018). Global action plan on physical activity 2018-2030. M ore active people for a healthier world. Recuperado de https:/ / www. who.int/ ncds/ prevention/ physicalactivity/ global-action-plan-2018-2030/ en/

O'Reilly, M., Dogra, N., Hughes, J., Reilly, P., George, R., \&W hiteman, N. (2018). Potential of social mediain promoting mental health in adolescents. $\mathrm{H}$ ealth Promotion International, 34(5), 981-991. http:// doi.org/ 10.1093/ heapro/ day056

0 viedo, H.C. y Campos-Arias, A. (2005). Aproxima ción al uso del coeficiente alfa de Cronbach. Revista colombiana de psiquiatría, 34(4), 572-580.

Pastor Ruiz,Y., M artín N ieto, R., \& M ontesVozmediano, M. (2019): Patrones de uso, control parental y acceso a la información de los adolescentes en la red. Estudios sobre el M ensaje Periodístico, 25(2), 995-1012.

Pérez, V. (2018). A proximación a la investigación psicológica en Internet y redes sociales. Summa Psicológica UST , 15(1), 98-105. http:/ / doi.org.10.18774/ 0719448x.2018.15.vp

Richmond, R. C. , Smith, G. D., Ness, A. R., den Hoed, M., M cM ahon, G., \&Timpson, N. J. (2014). Assessing causality in the association between child adiposity and physical activity levels: a Mendelian randomization analysis. PLOS M ed, 11(3), el001618. https:/ / doi.org/ 10.1371/ journal.pmed. 1001618

Rodríguez, A. N., García, A. R. R., \& Minguet, J. L. C. (2020). Los ambientes de aprendizaje como metodología activa promotora de la actividad física en Educación Infantil: un estudio de caso. Retos: nuevas tendencias en educación física, deporte y recreación, 37, 498-504.

Sallis, J. F. (1991). Self report measures of children's physical activity. J. School H ealth 61, 215-219.

Sánchez-Zafra, M., Ramirez-Granizo, I. A., Bæez-M irón, F., Moreno-Arrebola, R., Fernández-Revelles, A. B. (2019). Análisis de la relación existente entre el uso de videojuegos y la práctica de actividad física. Sportis Sci J, 5 (1), 118-132. https:/ / doi.org/ 10.17979/ sportis. 2019.5.1.3433

Stellefson, M., Paige, S. R. , Chaney, B. H., \& Chaney, J. D. (2020). Social M edia and Health Promotion. Int. J. Environ. Res. Public Health, 17(9), 3323. http:/ / doi.org/ 10.3390/ ijerph17093323
Tejada Garitan. E., Castaño Garrido, C., \& Romero Andonegui, A. (2019). Los hábitos de uso en las redes sociales de los preadolescentes. RIED. Revista Ibe roamericana de Educación a Distancia, 22(2), 119-133. http:/ / dx. doi.org/ 10.5944/ ried.22.2.23245

Twenge, J. M., Krizan, Z., \& Hisler, G. (2017). Decreases in self-reported sleep duration among US adolescents 2009-2015 and association with new media screen time. Sl eep medicine, 39, 47-53. https:/ / doi. org/ 10.1016/ j.sleep. 2017.08.013

Twenge, J. M., \& Campbell, W. K. (2018). Associations between screen time and lower psychological wellbeing among children and adolescents: Evidence from a population-based study. Preventive medicine reports, 12, 271-283. https:/ / doi.org/ 10.1016/ j. pmedr.2018.10.003

Van Woudenberg, T. J., Bevelander, K. E., Burk, W. J., Smit, C. R., Buijs, L., \& Buijzen, M. (2020). Testing a social network intervention using vlogs to promote physical activity among adolescents: a randomized controlled trial. Frontiers in psychology, 10, 2913. https:/ / doi. org/ 10.3389/ fpsyg. 2019.02913

Villanueva-Blasco, V. J., \& Serrano-Bernal, S. (2019). Patrón de uso de internet y control parental de redes sociales como predictor de sexting en adolescentes: una perspectiva de género. Revista de Psicología y Educación, 14(1), 16-26. https:/ / doi.org/ 10.23923/ rpye2019.01.168

Viner, R. M., Gireesh, A., Stiglic, N., Hudson, L. D., Goddings, A. L.,Ward, J. L. , \& Nicholls, D. E. (2019). Roles of cyberbullying, sleep, and physical activity in mediating the effects of social media use on mental health and wellbeing among young people in England: a secondary analysis of longitudinal data. The Lancet Child \& Adolescent H ealth, 3(10), 685-696.

Vergara-Torres, A. P., Juvera-Portilla, J. L., CeballosGurrola, 0., \& Zamarripa, J. (2020). Pokémon GO y su relación con la actividad física, orientación espacial y socialización en usuarios mexicanos. Retos: nuevastendencias en educación física, deportey recreación, 38, 727-732.

Zueck-Enríquez, M., Ramírez-García, A. A., RodríguezVillalobos, J. M., \& Irigoyen-Gutiérrez, H. E. (2020). Satisfacción en las clases de Educación Física y la intencionalidad de ser activo en niños del nivel de primaria. Retos: nuevas tendencias en educación física, deportey recreación, 37, 33-40. 